Research Article

\title{
Bamboo Rayon-ZnO Nanoparticles Composite as Multifunctional Textile Materials
}

\author{
Javed Sheikh and M. D. Teli \\ Department of Fibres and Textile Processing Technology, I.C.T., Mumbai 400019, India \\ Correspondence should be addressed to M. D. Teli; md.teli@ictmumbai.edu.in
}

Received 30 September 2013; Revised 24 January 2014; Accepted 3 February 2014; Published 10 March 2014

Academic Editor: Sambandam Anandan

Copyright ( $(2014$ J. Sheikh and M. D. Teli. This is an open access article distributed under the Creative Commons Attribution License, which permits unrestricted use, distribution, and reproduction in any medium, provided the original work is properly cited.

In the current study, an acrylic acid grafted bamboo rayon fabric was utilized as a substrate to immobilize ZnO nanoparticles. The bamboo rayon- $\mathrm{ZnO}$ nanoparticles composite was prepared by the treatment of swollen grafted fabric with $\mathrm{ZnCl}_{2}$ followed by conversion of $\mathrm{Zn}^{2+}$ ions into $\mathrm{ZnO}$ nanoparticles. The modified product was characterized and then evaluated for antibacterial activity against gram-positive and gram-negative bacteria as well as durability of their antibacterial activity after washing. The product showed antibacterial activity against both types of bacteria which was found to be durable till 40 washes. The modified material also showed improved UV protection. The product can be claimed as semidurable multifunctional textile material.

\section{Introduction}

With increase in awareness about health and the requirement of protection, a number of functional properties are expected from textile materials. Textiles can be colonized by microbes which can result in adverse effects both on textiles and users. Apart from this the UV protection is becoming one of the desirable properties as textiles act as barrier layer between human body and the environment and can protect human body from harmful effects on skin of UV light if finished properly. Bamboo, a lignocellulosic material, belonging to the grass family Poaceae, is an abundant renewable natural resource capable of production of maximum biomass per unit area and time as compared to counterpart timber species. Bamboo pulp fibre is widely applied in textile industry to produce dry goods. Generally, bamboo pulp fibre loses its natural antibacterial property present inherently in bamboo due to its treatment with alkali in its manufacturing process. The cellulosic fibre like bamboo rayon possesses many desirable properties which are suitable for apparels as well as medical textiles; however, the lack of antibacterial and UV protection properties is considered to be a severe limitation [1-8].

The grafted fibres especially from hydrophilic monomer like acrylic acid adsorb metal ions from the solution and also swell in contact with water. Hence they offer suitable substrate to immobilize the nanoparticles and to form nanoparticles composites. Incorporation of metal nanoparticles into polymer matrix has been reported by various researchers. Silver nanoparticles have been incorporated into poly(N-vinylpyrrolidone) (PVP) nanofibers using two distinct methods [9]. The hydrogel-silver nanocomposites have been synthesized by a unique methodology, which involves formation of silver nanoparticles within swollen poly(acrylamide-co-acrylic acid) hydrogels which showed efficient antibacterial activity against $E$. coli $[10]$. Researchers modified the cotton fibres by periodate oxidation followed by covalent attachment of chitosan and then loaded the modified fibre with copper ions and copper nanoparticles. These fibres showed an excellent antibacterial activity against E. coli [11]. The copper alginate-cotton cellulose (CACC) composite fibers and Cu nanoparticle loaded CACC fibres are reportedly found to display efficient antibacterial property [12]. Bajpai et al. prepared calcium alginate impregnated cotton fabric loaded with copper nanoparticles to impart antimicrobial properties [13]. Chaurasia et al. prepared $\mathrm{ZnO}$ nanoparticles loaded cellulose acetate (ZOLCA) films [14]. Bajpai et al. also prepared chitosan films loaded with $\mathrm{ZnO}$ nanoparticles for food packaging applications which have shown excellent antibacterial activity against the model bacterium Escherichia coli [15]. Zinc oxide nanoparticles loaded calcium alginate 


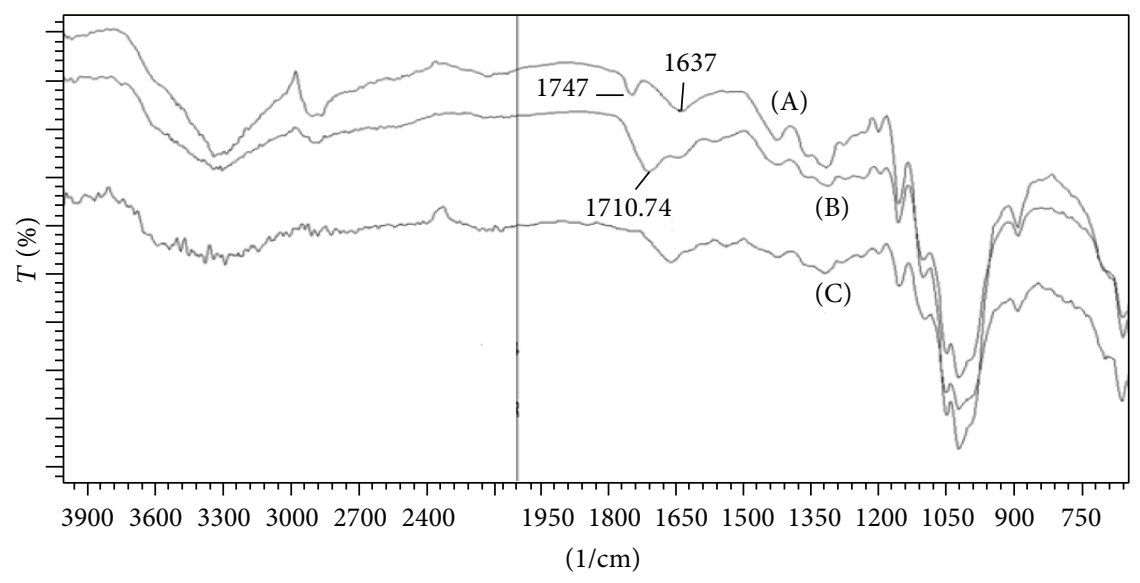
(A) Bamboo rayon (BR)
(B) AA-g-BR
(C) NanoZnO.AA-g-BR

FIGURE 1: FTIR spectra of fibres.

films are reportedly prepared for packaging purposes which are found to be biocidal against bacteria Escherichia coli [16].

The deployment of different nanoparticles on grafted bamboo rayon has been reported earlier from our laboratory [17-22]. Although a number of papers regarding antibacterial activity of $\mathrm{ZnO}$ have been reported, their application on textiles to get durable properties has been its limitation. In the present work, $\mathrm{ZnO}$ nanoparticles were immobilized on acrylic acid grafted bamboo rayon (AA-g-BR). The efficacy of such modified fabrics was analyzed and the durability of functional properties was also evaluated.

\section{Materials and Methods}

2.1. Materials. The bamboo rayon yarn was knitted to make fabric (single jersey, gsm-133.28, WPI-36, CPI-38) which was then scoured using $\mathrm{NaOH}(4 \%$ owf) and soda ash $(1 \%$ owf). The scoured fabric was used for grafting. All chemicals $\left(\mathrm{ZnCl}_{2}, \mathrm{NaOH}, \mathrm{K}_{2} \mathrm{~S}_{2} \mathrm{O}_{8}\right.$, and acrylic acid) used were of laboratory grade.

2.2. Methods. Grafting of acrylic acid onto bamboo rayon was carried out as per the procedure reported in our earlier research paper [23]. After completion of reaction, the grafted fabric was then washed with boiling water repeatedly, to remove the homopolymers of acrylic acid, till the constant weight was obtained indicating complete removal of homopolymer. The $\mathrm{ZnO}$ nanoparticle loaded grafted bamboo rayon fabric was prepared by using the procedure mentioned in the literature [24] with slight modification of curing $\mathrm{NaOH}$ treated fabric at $100^{\circ} \mathrm{C}$ for $2 \mathrm{~h}$. The composite fabric (nanoZnO-AA-g-BR) was characterized using FTIR, TGA, and SEM analysis. In order to study the effect of modification on the shade change of the fabric, the unmodified and composite bamboo rayon fabric samples were evaluated for the colour value using Spectraflash SF 300 supplied by Datacolor international. In order to estimate the stiffness of the fabric, its bending length was measured on Shirley stiffness tester. The
nanoZnO·AA-g-BR was evaluated for antibacterial activity using AATCC-100 [25]. The UV protection factor indicating the ability of the finished fabric to protect the wearers skin from UV rays and durability of such functional properties to washing was determined as explained in the literature [26].

\section{Results and Discussion}

3.1. Preparation of $\mathrm{ZnO}$ Nanoparticles Loaded Bamboo Rayon Fabric. When grafted bamboo rayon sample was put in water, it swells to some extent due to the hydrophilic nature of acrylic acid as well as bamboo rayon. The grafted fabric was further treated with $\mathrm{ZnCl}_{2}$, where the adsorption of zinc ions takes place and the adsorption mechanism can be viewed as complex formation of zinc ions with carboxylic groups. When the swollen fabric containing $\mathrm{Zn}^{2+}$ ions was put in sodium hydroxide solution and cured, the ions are converted to $\mathrm{ZnO}$ nanoparticles and distributed almost uniformly throughout the network.

3.2. Characterization of Modified Products. The grafted bamboo rayon fabric was characterized in order to validate grafting. The optimization of the grafting reaction was earlier reported from our laboratory [23]. The add-on (graft yield $\%)$ was found to be $14.85 \%$ with the reaction parameters mentioned in the experimental section. The FTIR spectrum of grafted fabric (refer to Figure 1) when compared with that of the ungrafted fabric clearly indicated the peak at $1711 \mathrm{~cm}^{-1}$ which is due to introduction of $-\mathrm{COOH}$ group as a part of graft side chains onto bamboo rayon substrate. Hardly any change in the FTIR spectra of AA-g-BR and nanoZnO-AA-g$\mathrm{BR}$ was observed as the nanoparticles were just immobilized on the grafted bamboo rayon and there was no introduction of any functional groups.

Figure 2 shows the thermograms of ungrafted bamboo rayon, $\mathrm{AA}-\mathrm{g}-\mathrm{BR}$, and nanoZnO-AA-g-BR samples. In the initial stage weight loss values of these samples were $9.5 \%$, $12 \%$, and $8.54 \%$ at $250^{\circ} \mathrm{C}$, respectively. Between $250^{\circ} \mathrm{C}$ and 
TABLE 1: Colour values of composite fabric (nanoZnO·AA-g-BR).

\begin{tabular}{lccccrrr}
\hline Sr. number & Nature of sample & $\begin{array}{c}\mathrm{ZnCl}_{2} \text { conc. } \\
\% \text { (owf) }\end{array}$ & Bending length $(\mathrm{cm})$ & $\mathrm{K} / \mathrm{S}$ & $\mathrm{L}^{*}$ & $\mathrm{a}^{*}$ & $\mathrm{~b}^{*}$ \\
\hline 1 & Ungrafted & 0 & 2.35 & 0.2229 & 82.59 & -0.84 & 8.42 \\
2 & AA-g-BR & 0.25 & 2.70 & 0.2000 & 88.72 & 0.64 & 8.84 \\
3 & AA-g BR & 0.5 & 2.75 & 0.3055 & 85.22 & 0.57 & 9.90 \\
4 & AA-g BR & 1.0 & 2.75 & 0.3143 & 82.94 & 0.52 & 7.90 \\
\hline
\end{tabular}

$\mathrm{L}^{*}, \mathrm{a}^{*}$ and $\mathrm{b}^{*}$ are the colour co-ordinates.

TABLE 2: Effect of $\mathrm{ZnCl}_{2}$ concentration on antibacterial properties of nanoZnO·AA-g-BR.

\begin{tabular}{lcccc}
\hline \multirow{2}{*}{ Sr. number } & Nature of sample & \multicolumn{2}{c}{$\mathrm{ZnCl}_{2}$ conc. } & \multicolumn{2}{c}{ Bacterial reduction (\%) } \\
\hline 1 & Ungrafted & 0 & $\mathrm{~N}$ & $\mathrm{~N}$ \\
2 & AA-g-BR & 0 & 35.52 & 30.35 \\
3 & AA-g-BR & 0.25 & 88.60 & 89.78 \\
4 & AA-g-BR & 0.5 & 100 & 98.13 \\
5 & AA-g-BR & 1.0 & 100 & 99.56 \\
\hline
\end{tabular}

N: negligible.

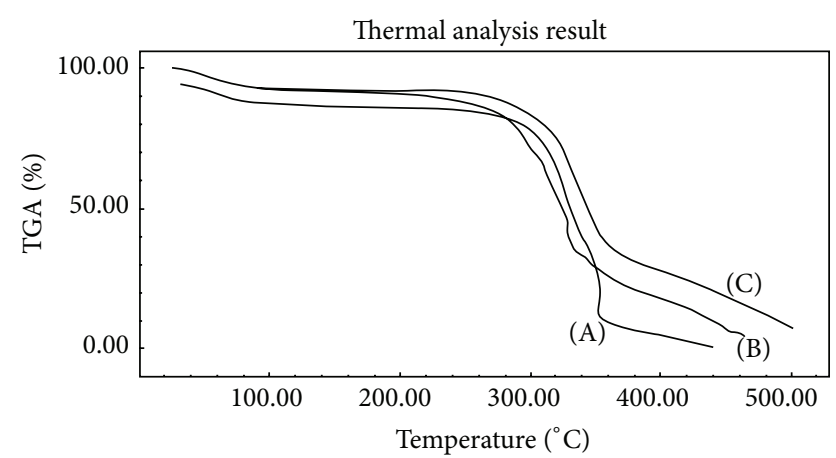

(A) Bamboo rayon (BR)

(B) AA-g-BR

(C) NanoZnO.AA-g-BR

FIGURE 2: TGA of bamboo rayon, AA-g-BR, and nanoZnO·AA-gBR.

$350^{\circ} \mathrm{C}$, the drastic decomposition of the samples resulted in significant weight loss which was $59.22 \%$ for ungrafted, $56.2 \%$ for AA-g-BR, and $46.89 \%$ for nanoZnO-AA-g-BR fabrics at $350^{\circ} \mathrm{C}$

However, beyond $350^{\circ} \mathrm{C}$, the loss in weight was slowed down and finally at $450^{\circ} \mathrm{C}$, weight loss values observed were $96.81 \%$ for ungrafted, $90.13 \%$ for AA-g-BR, and $80.95 \%$ for nanoZnO-AA-g-BR. This clearly indicates the higher thermal stability of AA-g-BR as compared to that of ungrafted bamboo rayon. The composite fabric (nano $\mathrm{ZnO} \cdot \mathrm{AA}-\mathrm{g}-\mathrm{BR}$ ) also showed further enhancement in thermal stability as compared to that of ungrafted bamboo rayon and AA-gBR. This could be attributed to the formation of side chain network as a result of grafting of acrylic acid onto bamboo rayon substrate increasing molecular weight and due to immobilization of immobilized $\mathrm{ZnO}$ nanoparticles in the polyacrylic acid matrix.
The surface morphology of the nano $\mathrm{ZnO} \cdot \mathrm{AA}-\mathrm{g}-\mathrm{BR}$ containing grafted bamboo fabric was studied using SEM analysis. The images of the fabric surfaces are shown in Figure 3 which clearly indicate the presence of $\mathrm{ZnO}$ nanoparticles on the surface of the modified fabric and these nanoparticles are visible even at a low magnification. Some agglomeration of the $\mathrm{ZnO}$ nanoparticles was also observed.

3.3. Change in Appearance and Stiffness of the Modified Fabric. The change in appearance of modified fabrics due to nanoZnO formation was measured (refer to Table 1) on Spectrophotometer.

The K/S values were of very small order, indicating negligible change in appearance of the fabric. $\mathrm{ZnO}$ nanoparticles are white in colour and hence offer the advantage in terms of minimal change in appearance. The bending length increased after modification indicating the increase in stiffness of modified bamboo rayon. However, the increase in bending length with the concentration of $\mathrm{ZnCl}_{2}$ was insignificant.

3.4. Antibacterial Activity and Durability of the Modified Fabrics. The quantitative antibacterial assessment was made using AATCC-100 (2004) test method and the results are presented in Tables 2 and 3 .

The bamboo rayon fabric showed no antibacterial activity against both $S$. aureus and $E$. coli as reported by some researchers [17-22]. The results clearly indicate the excellent antibacterial activity of the nanoZnO-AA-g-BR samples which was found to be improving with increase in concentration of $\mathrm{ZnCl}_{2}$. This was quite obvious as the antibacterial action was because of $\mathrm{ZnO}$ nanoparticles which will be in the higher concentration when higher concentration of $\mathrm{ZnCl}_{2}$ was taken. At $0.5 \% \mathrm{ZnCl}_{2}$, antibacterial activity against $S$. aureus was $100 \%$ whereas that against E. coli was $98.13 \%$. Further increase in concentration of $\mathrm{ZnCI}_{2}$ to $1 \%$ did not show appreciable increase in antibacterial properties against 
TABLE 3: Durability of the antibacterial properties of ungrafted and grafted samples loaded with nanoZnO $(0.5 \%)$.

\begin{tabular}{|c|c|c|c|c|c|}
\hline \multirow{3}{*}{ Sr. number } & \multirow{3}{*}{ Number of washes } & \multicolumn{4}{|c|}{ Bacterial reduction (\%) } \\
\hline & & \multicolumn{2}{|c|}{ Ungrafted } & \multicolumn{2}{|c|}{ Grafted } \\
\hline & & S. aureus & E. coli & S. aureus & E. coli \\
\hline 1 & 0 & 87.52 & 87.90 & 100 & 98.13 \\
\hline 2 & 5 & 41.95 & 43.10 & 92.88 & 90.04 \\
\hline 3 & 10 & 18.25 & 19.10 & 82.19 & 82.93 \\
\hline 4 & 20 & - & - & 78.63 & 79.02 \\
\hline 5 & 40 & - & - & 71.36 & 72.98 \\
\hline 6 & 50 & - & - & 56.98 & 60.18 \\
\hline
\end{tabular}

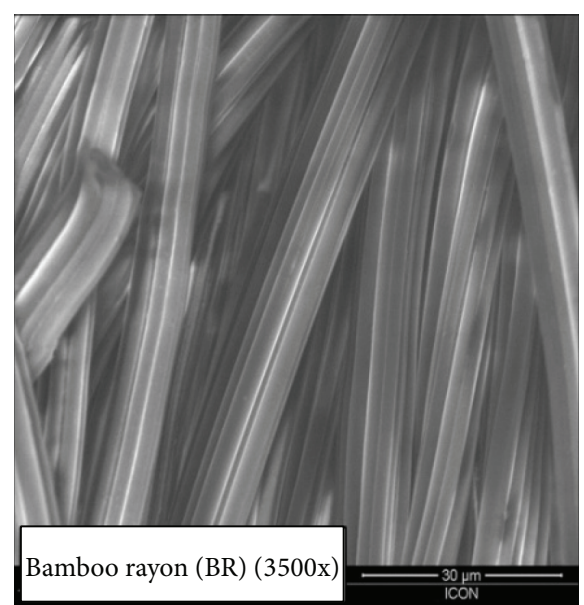

(a)

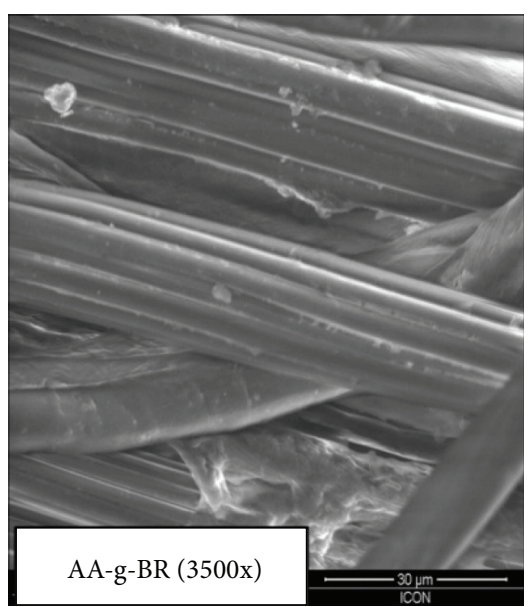

(b)

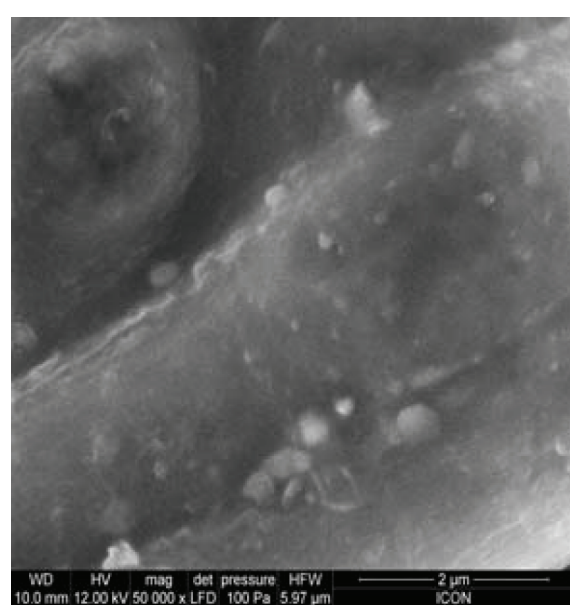

(c)

FIGURE 3: SEM photographs of ungrafted (a) bamboo rayon, AA-g-BR (b), and nanoZnO-AA-g-BR (c).

TABLE 4: Durability of the ultraviolet protection of nanoZnO·AA-gBR $(0.5 \%)$

\begin{tabular}{lcc}
\hline Sr. number & Number of washes & Calculated UPF \\
\hline 1 & $\mathbf{0}$ & 106 \\
2 & $\mathbf{5}$ & 75 \\
3 & $\mathbf{1 0}$ & 55 \\
4 & $\mathbf{2 0}$ & 46 \\
5 & $\mathbf{3 0}$ & 21 \\
\hline
\end{tabular}

E. coli and hence the optimum concentration for antibacterial property was taken as $0.5 \% \mathrm{ZnCl}_{2}$. The similar trends were observed in our earlier study [22].

In case of $\mathrm{ZnO}$ nanoparticle containing ungrafted bamboo rayon, the unwashed sample showed excellent antibacterial activity against both Gram-positive and Gram-negative bacteria. However, the antibacterial activity was reduced drastically after subsequent washing showing only $50 \%$ reduction after 5 washes. The nanoZnO-AA-g-BR showed reduction of $S$. aureus from $100 \%$ to $71.36 \%$ even after 40 washes (refer to Table 3). In case of E. coli the reduction was from $98.13 \%$ for freshly modified to $72.98 \%$ after 40 washes. The results clearly indicate the stronger holding of $\mathrm{ZnO}$ nanoparticles by bamboo rayon fabric grafted with acrylic acid. This may be attributed to introduction of side chains of acrylic acid on the bamboo rayon backbone during grafting which leads to better interaction of such substrates with $\mathrm{ZnO}$ nanoparticles. The results emphasize that the antibacterial activity of nanoZnO-AA-g-BR fabric was very intense even with small amount of $\mathrm{ZnO}$ nanoparticles. The excellent fastness to washing is due to antibacterial agent being immobilized.

The UV protection of the modified fabrics was evaluated and the results are summarized in Table 4, which indicate the efficient UV protection by the nanoZnO-AA-g-BR. In general UV protection factor (UPF) in excess of 50 is considered to be an excellent protection. The UPF was found to be decreasing with subsequent washing showing the value 46 , that is, close to be excellent after 20 washes. Hence it can be claimed that the modified fabric could display efficient UV protection till at least 20 washes.

\section{Conclusion}

Bamboo Rayon-ZnO nanoparticles composites were successfully prepared using acrylic acid grafted bamboo rayon substrate. The acrylic acid graft chains were responsible for immobilization of $\mathrm{ZnO}$ nanoparticles. Due to this type of anchoring the modified product displayed efficient and durable antibacterial activity and semidurable UV protection. 


\section{Conflict of Interests}

The authors do not have any stake in Datacolor International or AATCC and their names appear in the paper purely in coincidental and academic nature. Their reference is made to indicate standard equipment and test method made use of in this work and in no way it advocates these brands.

\section{Acknowledgment}

The authors gratefully acknowledge University Grants Commission (UGC-SAP) India for the research fellowship.

\section{References}

[1] Y. Gao and R. Cranston, "Recent advances in antimicrobial treatments of textiles," Textile Research Journal, vol. 78, no. 1, pp. 60-72, 2008.

[2] W. Su, S. S. Wei, S. Q. Hu, and J. X. Tang, "Antimicrobial finishing of cotton textile with nanosized silver colloids synthesized using polyethylene glycol," Journal of the Textile Institute, vol. 102, no. 2, pp. 150-156, 2011.

[3] G. Sun, X. Xu, J. R. Bickett, and J. F. Williams, "Durable and regenerable antibacterial finishing of fabrics with a new hydantoin derivative," Industrial and Engineering Chemistry Research, vol. 40, no. 4, pp. 1016-1021, 2001.

[4] T. Nakashima, Y. Sakagami, H. Ito, and M. Matsuo, "Antibacterial activity of cellulose fabrics modified with metallic salts," Textile Research Journal, vol. 71, no. 8, pp. 688-694, 2001.

[5] M. Joshi, S. W. Ali, R. Purwar, and S. Rajendran, "Ecofriendly antimicrobial finishing of textiles using bioactive agents based on natural products," Indian Journal of Fibre and Textile Research, vol. 34, no. 3, pp. 295-304, 2009.

[6] K. C. Lin, Y. S. Wu, T. H. Tan, and T. L. Tai, "Graft copolymerization of acrylonitrile onto bamboo using IR spectroscopic technique as a probe," in Graft Copolymerization of Lignocellulosic Fibers, D. N. S. Hon, Ed., ACS Symposium Series 187, pp. 233250, American Chemical Society, Washington, DC, USA, 1982.

[7] R. Sharma, V. K. Varshney, G. S. Chauhan, S. Naithani, and P. L. Soni, "Hydroxypropylation of cellulose isolated from bamboo (Dendrocalamus strictus) with respect to hydroxypropoxyl content and rheological behavior of the hydroxypropyl cellulose," Journal of Applied Polymer Science, vol. 113, no. 4, pp. 2450-2455, 2009.

[8] Z. Qin, Y. Chen, P. Zhang, G. Zhang, and Y. Liu, "Structure and properties of $\mathrm{Cu}(\mathrm{II})$ complex bamboo pulp fabrics," Journal of Applied Polymer Science, vol. 117, no. 3, pp. 1843-1850, 2010.

[9] W. Jin, H. K. Lee, R. H. Jeong, W. H. Park, and J. H. Youk, "Preparation of polymer nanofibers containing silver nanoparticles by using poly(N-vinylpyrrolidone)," Macromolecular Rapid Communications, vol. 26, no. 24, pp. 1903-1907, 2005.

[10] V. Thomas, M. M. Yallapu, B. Sreedhar, and S. K. Bajpai, "A versatile strategy to fabricate hydrogel-silver nanocomposites and investigation of their antimicrobial activity," Journal of Colloid and Interface Science, vol. 315, no. 1, pp. 389-395, 2007.

[11] G. Mary, S. K. Bajpai, and N. Chand, "Copper (II) ions and copper nanoparticles-loaded chemically modified cotton cellulose fibers with fair antibacterial properties," Journal of Applied Polymer Science, vol. 113, no. 2, pp. 757-766, 2009.

[12] M. Grace, N. Chand, and S. K. Bajpai, "Copper alginate-cotton cellulose (CACC) fibers with excellent antibacterial properties,"
Journal of Engineered Fibers and Fabrics, vol. 4, no. 3, pp. 24-35, 2009.

[13] S. K. Bajpai, M. Bajpai, and L. Sharma, "Copper nanoparticles loaded alginate-impregnated cotton fabric with antibacterial properties," Journal of Applied Polymer Science, vol. 126, supplement 1, pp. E319-E326, 2012.

[14] V. Chaurasia, N. Chand, and S. K. Bajpai, "Water sorption properties and antimicrobial action of zinc oxide nanoparticlesloaded cellulose acetate films," Journal of Macromolecular Science A: Pure and Applied Chemistry, vol. 47, no. 4, pp. 309-317, 2010.

[15] S. K. Bajpai, N. Chand, and V. Chaurasia, "Investigation of water vapor permeability and antimicrobial property of Zinc Oxide nanoparticles-loaded chitosan-based edible film," Journal of Applied Polymer Science, vol. 115, no. 2, pp. 674-683, 2010.

[16] S. K. Bajpai, N. Chand, and V. Chaurasia, "Nano zinc oxideloaded calcium alginate films with potential antibacterial properties," Food and Bioprocess Technology, vol. 5, no. 5, pp. 18711881, 2012.

[17] M. D. Teli and J. Sheikh, "Antibacterial and acid and cationic dyeable bamboo rayon on grafting," Carbohydrate Polymers, vol. 88, no. 4, pp. 1281-1287, 2012.

[18] M. D. Teli and J. Sheikh, "Nanosilver containing bamboo rayon as durable antibacterial material," Fibers and Polymers, vol. 13, no. 10, pp. 1280-1285, 2012.

[19] M. D. Teli and J. Sheikh, "Study of grafted silver nanoparticle containing durable antibacterial bamboo rayon," Cellulose Chemistry and Technology, vol. 47, no. 1-2, pp. 69-75, 2013.

[20] M. D. Teli and J. Sheikh, "Modified Bamboo rayon-Copper nanoparticle composites as durable antibacterial textiles," International Journal of Biological Macromolecules, vol. 61, pp. 302307, 2013.

[21] M. D. Teli and J. Sheikh, "Bamboo rayon-Copper nanoparticle composites as durable antibacterial textile materials," Composite Interfaces, vol. 21, no. 2, pp. 161-171, 2014.

[22] J. Sheikh and M. D. Teli, "Functional Modication of Bamboo Rayon (Cellulose) fabric to render it antibacterial and UV protective," Advanced Material Research, vol. 747, pp. 509-513, 2013.

[23] M. D. Teli and J. Sheikh, "Grafting of bamboo rayon with acrylic acid and its effect on cationic dyeing," Cellulose Chemistry and Technology, vol. 46, no. 1-2, pp. 53-59, 2012.

[24] S. K. Bajpai, V. Thomas, and M. Bajpai, "Novel strategy for synthesis of $\mathrm{ZnO}$ microparticles loaded cotton fabrics and Investigation of their antibacterial properties," Journal of Engineered Fibers and Fabrics, vol. 6, no. 3, pp. 73-81, 2011.

[25] American Association of Textile Chemists and Colorists, AATCC Technical Manual, vol. 76, AATCC, Research Triangle Park, NC, USA, 2007.

[26] ISO Technical Manual, Geneva, Switzerland, 2006. 

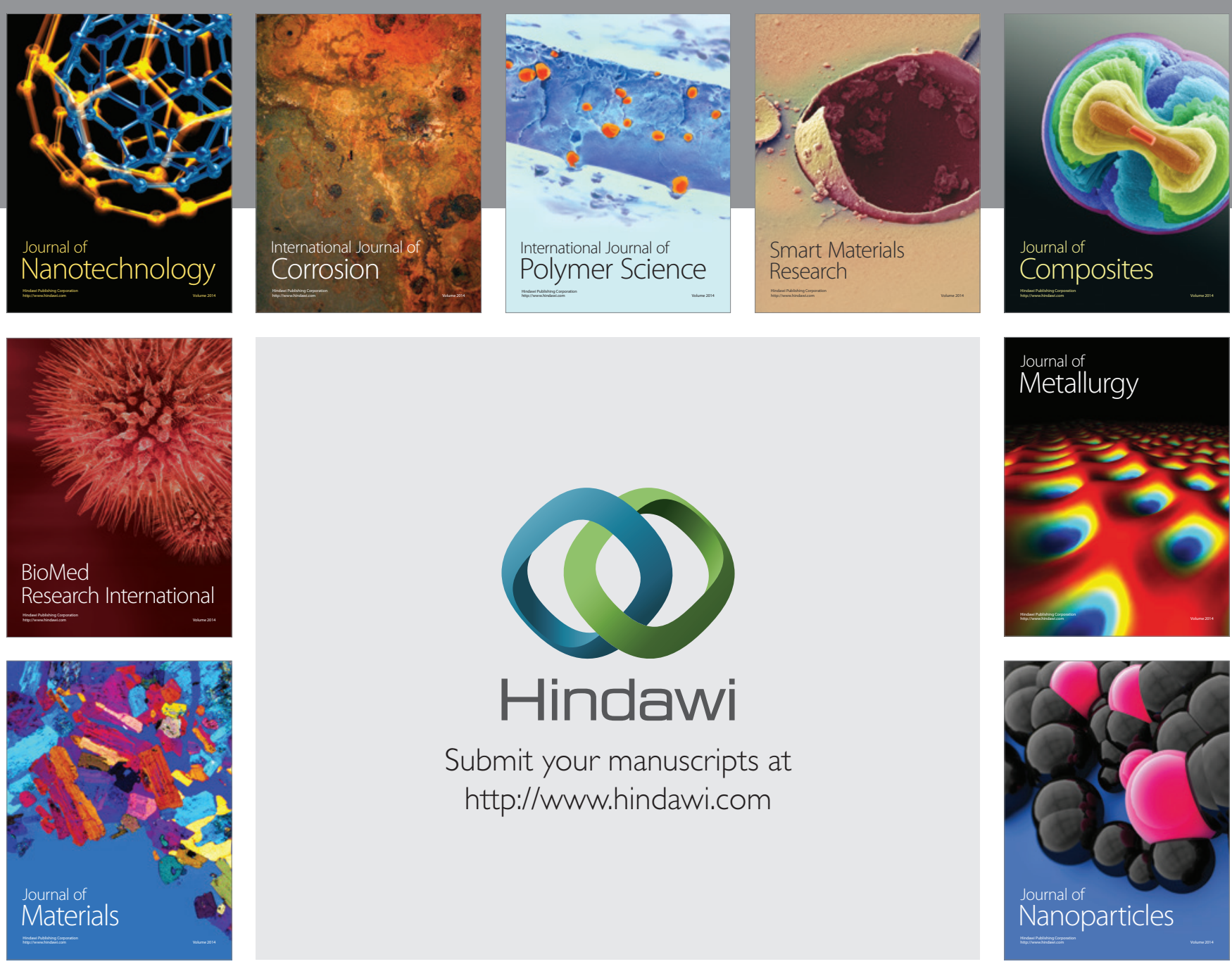

Submit your manuscripts at http://www.hindawi.com
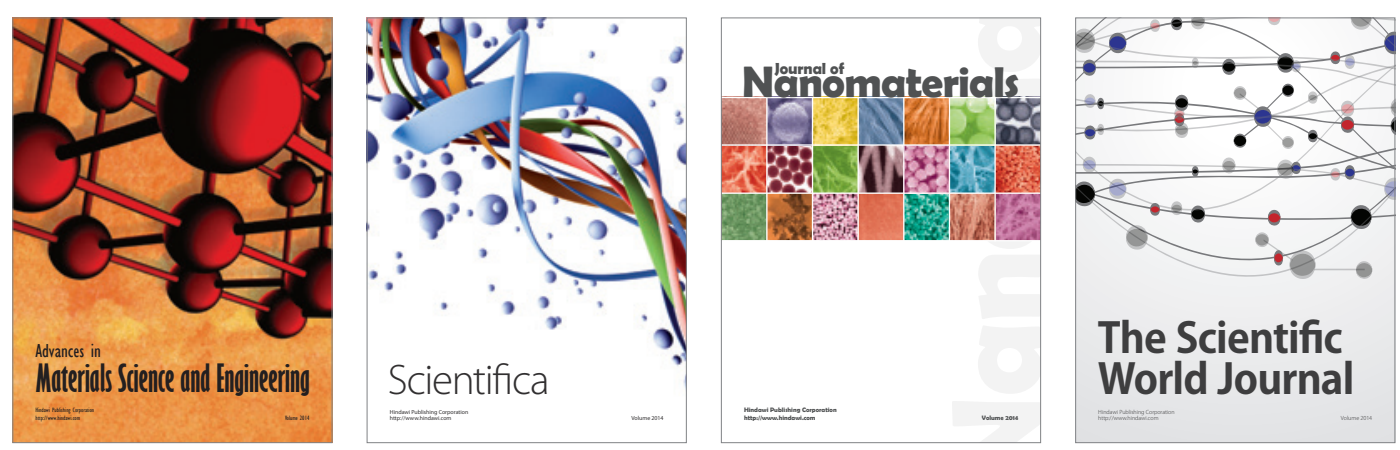

\section{The Scientific World Journal}
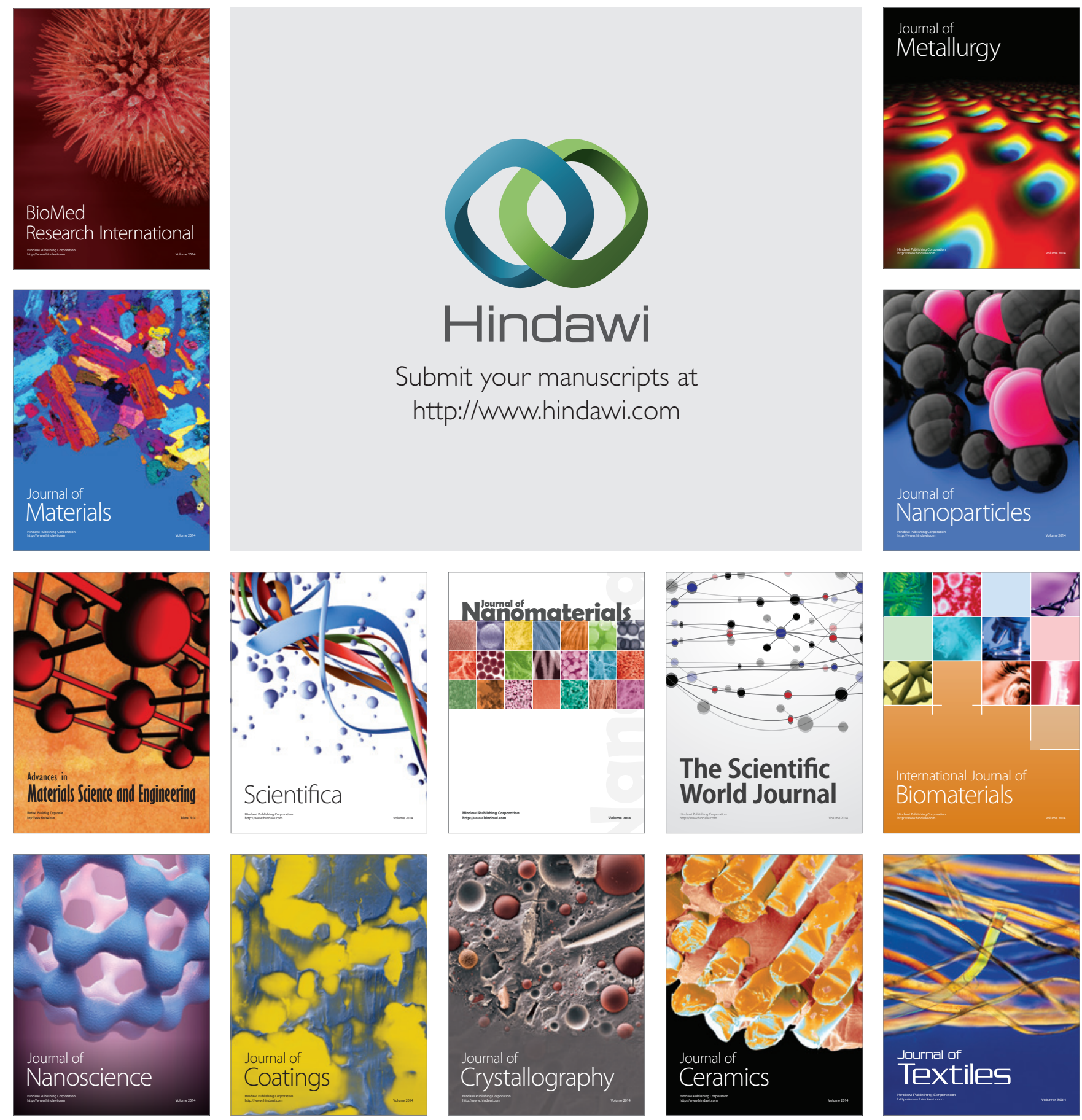